\title{
The Road Ahead
}

\author{
Kathy Zebracki, PhD, ${ }^{1,2}$ and Lawrence C. Vogel, $M D^{1,3}$ \\ ${ }^{1}$ Shriners Hospitals for Children, Chicago, Illinois; ${ }^{2}$ Northwestern University Feinberg School of Medicine, Chicago, Illinois; \\ ${ }^{3}$ Rush Medical College, Chicago, Illinois
}

$\mathrm{I}$

$\mathrm{t}$ is an enormous privilege to be the new Editors-in-Chief of Topics in Spinal Cord Injury Rehabilitation (TSCIR). After 4.5 years of tireless contributions, James S. Krause, PhD, has elected to step down. We are very grateful to Dr. Krause and his editorial team for handing over a vibrant journal. During his tenure, TSCIR has progressed in many ways, including increasing the page count per issue, widening the expert reviewer base, and undoubtedly, most excitingly, producing high-quality content such that TSCIR is now indexed by leading databases such as Index Medicus, MEDLINE, and PubMed Central. We are very pleased that Deborah Backus, $\mathrm{PhD}, \mathrm{PT}$ and M.J. Mulcahey, $\mathrm{PhD}$, OTR will remain as Associate Editors. We are also fortunate that Mary M. Killion will continue to serve as the Managing Editor for the journal.

We hope to retain the strong tradition of TSCIR by serving as the leading source for clinical, peerreviewed, and state-of-the art information on traumatic and nontraumatic spinal cord injury. We also request our readers help in growing TSCIR in new directions and expanding our utility and impact on the larger fields of spinal cord dysfunction, rehabilitation, and habilitation. As such, we propose 7 goals to achieve during our tenure.

First, a unique aspect and draw to TSCIR is its in-depth coverage of a single topic area per issue. We will address the variety of interests of our readers by including 2 key topical areas followed by proffered articles in most issues. We hope that this will encourage readers to peruse all articles in an issue as well as improve the time to publication once an article has been accepted.

Second, we would like to grow our readership by expanding our content area and encouraging submissions on diseases and conditions, such as nontraumatic SCI, multiple sclerosis, spina bifida, and brachial plexus injury.

Third, with the support and partnership of Spinal Cord Injury Model System and the Paralyzed Veterans of America, we will begin publishing fact sheets regarding various domains of spinal cord dysfunctions as well as clinical practical guidelines. Consequently, to support this goal, we encourage authors to submit systematic reviews (rigorous high-quality comprehensive review) and topical reviews (brief articles that summarize findings).

Fourth, we plan to develop a Junior Reviewing Mentoring program to support and train early career professionals regarding the editorial process. This will help create the next generation of outstanding reviewers and ensure the continuation of our high-quality review process.

Fifth, as TSCIR has recently been indexed, we would like to enhance the impact factor of the journal. We will work closely with Thomas Land Publishers to monitor our impact factor and the articles that are most frequently cited by and of most interest to others.

Sixth, we would like to expand the international audience for TSCIR and foster an increasing number of international authors. International SCI clinicians and scientists have a great deal of information to share that will further the science 
and ultimately benefit individuals with SCI from around the world.

Finally, this is your journal. We, along with the Editorial Board, will work assiduously to meet your clinical and research needs. We will do our best to publish clinically relevant research of the highest quality. If you have ideas for future topics or have a comment about something we are doing, please let us know! You may contact us at scieditor@thomasland.com.
With his expertise, generosity, and humor, Dr. Krause has been a true leader for TSCIR and will remain an invaluable asset to the journal. His is a hard act to follow, but we realize the road ahead is full of adventure, new knowledge, and exciting opportunities, and with your help, dedication to science, and hard work, we can carry on his legacy and bring TSCIR to new heights. 